\title{
Solution structure, stability and transitions of non-isothermal flow through a curved rectangular duct
}

\section{Maths. Discipline; Science, Engg. \& Technol. School, Khulna University, Khulna-9208, Bangladesh \\ R. N. Mondal, N. Islam, L. E. Ali and A. K. Datta rnmondal71@yahoo.com}

Abstract: In this paper, a numerical study is presented for the fully developed two-dimensional flow of viscous incompressible fluid through a curved rectangular duct. The outer wall of the duct is heated while the inner one is cooled. Numerical calculations are carried out by using the spectral method covering a wide range of the Dean number, $D_{n}, \quad 0 \leq D_{n} \leq 1500$ and the curvature, $\delta$, $0<\delta \leq 0.5$ for the Grashof number $\mathrm{Gr}=100$. First, steady solutions are obtained by the Newton-Raphson iteration method. As a result, six branches of symmetric steady solutions are obtained with two- and multi-vortex solutions. Its linear stability is then investigated. In the unstable region, time evolution calculations as well as their spectral analyses are performed. A typical transition occurs from steady flow to chaos through periodic or multi-periodic flows, if $D_{n}$ is increased no matter what $\delta$

is. Effects of curvature on the unsteady solutions are also obtained. The transition to the periodic or the chaotic state is retarded, if the curvature is increased.

Keywords: Curved rectangular duct, solution flow.

AMS Mathematics Subject Classification: 76D99, 76E09 Introduction

The flow through a curved duct has attracted considerable attention because of its physically and mathematically interesting feature under the action of a centrifugal force caused by the curvature of the duct. A secondary flow is induced by imbalance between the centrifugal force and the pressure gradient in a cross section of the duct. There have been a lot of theoretical and experimental works concerning this flow, here the review articles by Berger et al. (1983), Nandakumar and Masliyah (1986) and Ito (1987) may be referred to for some outstanding reviews on curved duct flows.

One of the interesting phenomena of the flow through a curved duct is the bifurcation of the flow because generally there exist many steady solutions due to channel curvature. Dennis and $\mathrm{Ng}$ (1982) and later Yanase, Gotoh and Yamamoto (1989) studied dual solutions of the flow through a curved duct. Yang and Keller (1986) studied the bifurcation of the flow for small curvature and found multiple branches of solutions. However, detailed bifurcation structures and linear stability of the steady solutions for fully developed flows in a curved square duct were investigated by Winters (1987) and by Mondal et al. (2007).

Time-dependent analysis of fully developed flows was initiated by Yanase and Nishiyama (1988) for a rectangular cross section in connection with the bifurcation diagram of steady solutions. In that study they investigated unsteady solutions for the case where dual solutions exist. The time-dependent behavior of the flow in a curved rectangular duct of large aspect ratio was

investigated, in detail, by Yanase et al. (2002) numerically. Recently, Wang and Yang (2005) performed numerical as well as experimental investigations of periodic oscillations for the fully developed flow in a curved square duct. They showed, both experimentally and numerically, that a temporal oscillation takes place between the symmetric/asymmetric two-vortex and fourvortex flows where there are no stable steady solutions. Very recently, Mondal et al. (2007) performed numerical prediction of the unsteady solutions through a curved square duct flow and showed that the steady flow turns into chaos through periodic or multi-periodic flows if the Dean number is increased.

Studies of isothermal flows through a curved duct for small curvature have been made, experimentally or numerically, for various shapes of the cross section by many authors. However, some tubes could have large curvature in practice which makes the action of the centrifugal force large and the flow characteristics more complicated. Yanase et al. (1994) numerically studied the effect of curvature on dual solutions for the flow through a curved duct of circular cross section. They also found that the four-vortex solution remains unstable even if the curvature is increased. Chandratilleke and Nursubyakto (2003) presented numerical calculations to describe the secondary flow characteristics in the flow through curved rectangular ducts of aspect ratios ranging from 1 to 8 that were heated on the outer wall, where they studied for small Dean numbers and compared the numerical results with their experimental data. Recently, Yanase et al. (2005) performed numerical investigation of isothermal and non-isothermal flows through a curved rectangular duct where they obtained many branches of steady solutions. Very recently, Mondal et al. (2006) performed numerical investigation of non isothermal flows in a curved square duct and studied the effects of curvature on the flow characteristics. But from the scientific as well as engineering point of view, it is quite interesting to study the transitional phenomena of the flow in a curved duct with the variation of curvature because this type of flow is often encountered in engineering applications.

In the present paper, numerical results are presented for the non-isothermal flow through a curved rectangular duct over a wide range of the Dean number and the curvature by finding steady solutions, investigating their linear stability and analyzing nonlinear time evolutions with spectral analysis of the unsteady solutions. Studying the transitional behavior of the unsteady solutions is an important objective of the present paper.

Mathematical formulations

Consider an incompressible viscous fluid streaming through a curved duct with rectangular cross section whose width and height are $2 d$ and $2 h$, respectively. The

Research article

(CIndian Society for Education and Environment (iSee)
"Numerical analysis of flow in curved duct" http://www.indjst.org
Mondal et al. Indian J.Sci.Technol. 
coordinate system with the relevant notations are shown in Fig. 1. It is assumed that the outer wall of the duct is heated while the inner one is cooled. The temperature of the outer wall is $T_{0}+\Delta T$ and that of the inner wall is $T_{0}-\Delta T$, where $\Delta T>0$. The $x, y$, and $z$ axes are taken to be in the horizontal, vertical, and axial directions, respectively. It is assumed that the flow is uniform in the axial direction, and that it is driven by a constant pressure gradient $G$ along the center-line of the duct, i.e. the main flow in the axial direction as shown in Fig. 1.

The variables are non-dimensionalized by using the representative length $d$ and the representative velocity $U_{0}=v / d$. We introduce the non-dimensional variables defined as:

$u=\frac{u^{\prime}}{U_{0}}, v=\frac{v^{\prime}}{U_{0}}, w=\frac{\sqrt{2 \delta}}{U_{0}} w^{\prime}, x=\frac{x^{\prime}}{d}, \bar{y}=\frac{y^{\prime}}{d}, \quad z=\frac{z^{\prime}}{d}$ $T=\frac{T^{\prime}}{\Delta T}, t=\frac{U_{0}}{d} t^{\prime}, \quad \delta=\frac{d}{L}, \quad P=\frac{P^{\prime}}{\rho U_{0}^{2}}$

where $u, v$, and $w$ are the non-dimensional velocity components in the $x, y$, and $z$ directions, respectively; $t$ is the non-dimensional time, $P$ the non-dimensional pressure, $\delta$ the non-dimensional curvature, and temperature is non-dimensionalized by $\Delta T$. Henceforth, all the variables are nondimensionalized if not specified. The sectional stream function $\psi$ is introduced as

$u=\frac{1}{1+\delta x} \frac{\partial \psi}{\partial \bar{y}}, v=-\frac{1}{1+\delta x} \frac{\partial \psi}{\partial x}$.

A new coordinate variable $y^{\prime}$ is introduced in the $y$ direction as $y=l y^{\prime}$, where $l=h / d$ is the aspect ratio of the duct cross section. From now on $y$ denotes $y^{\prime}$ for the sake of simplicity. Then the basic equations for $w, \psi$ and $T$ are derived from the Navier-Stokes equations and the energy equation under the Boussinesq approximation as,

$(1+\delta x) \frac{\partial w}{\partial t}+\frac{1}{l} \frac{\partial(w, \psi)}{\partial(x, y)}-D_{n}+\frac{\delta^{2} w}{1+\delta x}=(1+\delta x) \Delta_{2} w-\frac{\delta}{l(1+\delta x)} \frac{\partial \psi}{\partial y} w+\delta \frac{\partial w}{\partial x}$

$$
\begin{aligned}
& \left(\Delta_{2}-\frac{\delta}{1+\delta x} \frac{\partial}{\partial x}\right) \frac{\partial \psi}{\partial t}=-\frac{1}{l(1+\delta x)} \frac{\partial\left(\Delta_{2} \psi, \psi\right)}{\partial(x, y)}+\frac{\delta}{l(1+\delta x)^{2}} \\
& \times\left[\frac{\partial \psi}{\partial y}\left(2 \Delta_{2} \psi-\frac{3 \delta}{1+\delta x} \frac{\partial \psi}{\partial x}+\frac{\partial^{2} \psi}{\partial x^{2}}\right)-\frac{\partial \psi}{\partial x} \frac{\partial^{2} \psi}{\partial x \partial y}\right]+\frac{\delta}{(1+\delta x)^{2}} \\
& \times\left[3 \delta \frac{\delta^{2} \psi}{\partial x^{2}}-\frac{3 \delta^{2}}{1+\delta x} \frac{\partial \psi}{\partial x}\right]-\frac{2 \delta}{1+\delta x} \frac{\partial}{\partial x} \Delta_{2} \psi+\frac{1}{l} w \frac{\partial w}{\partial y} \\
& \times \Delta_{2}^{2} \psi-G r(1+\delta x) \frac{\partial T}{\partial x}
\end{aligned}
$$

$$
\frac{\partial T}{\partial t}+\frac{1}{l(1+\delta x)} \frac{\partial(T, \psi)}{\partial(x, y)}=\frac{1}{\operatorname{Pr}}\left(\Delta_{2} T+\frac{\delta}{1+\delta x} \frac{\partial T}{\partial x}\right) .
$$

ISSN: 0974- 6846

where

$\Delta_{2} \equiv \frac{\partial^{2}}{\partial x^{2}}+\frac{1}{l^{2}} \frac{\partial^{2}}{\partial y^{2}}, \frac{\partial(f, g)}{\partial(x, y)} \equiv \frac{\partial f}{\partial x} \frac{\partial g}{\partial y}-\frac{\partial f}{\partial y} \frac{\partial g}{\partial x}$.

The Dean number $D_{n}$, the Grashof number $G_{r}$, and the Prandtl number $P_{r}$, which appear in Eqs. (2) to (4) are defined as

$D_{n}=\frac{G d^{3}}{\mu \nu} \sqrt{\frac{2 d}{L}}, \quad G_{r}=\frac{\beta g \Delta T d^{3}}{v^{2}}, \quad P_{r}=\frac{v}{k}$

where $\mu, v, k$ and $g$ are the viscosity, the coefficient of thermal expansion, the coefficient of thermal diffusivity and the gravitational acceleration respectively.

The rigid boundary conditions for $w$ and $\psi$ are used as

$w( \pm 1, y)=w(x, \pm 1)=\psi( \pm 1, y)=\psi(x, \pm 1)=\frac{\partial \psi}{\partial x}( \pm 1, y)=\frac{\partial \psi}{\partial y}(x, \pm 1)=0$

and the temperature $T$ is assumed to be constant on the walls as

$T(1, y)=1, T(-1, y)=-1, T(x, \pm 1)=x$.

In the present study, $D_{n}$ and $\delta$ are varied while $l, G_{r}$ and $P_{r}$ are fixed as $l=2, G_{r}=100, \quad$ and $P_{r}=7.0$ (water).

\section{Numerical calculations}

In order to solve the Eqs. (2) to (4) numerically the spectral method is used. This is the method which is thought to be the best numerical method to solve the Navier-Stokes equations as well as the energy equation. By this method the variables are expanded in a series of functions consisting of the Chebyshev polynomials. That is, the expansion functions

$$
\phi_{n}(x)=\left(1-x^{2}\right) C_{n}(x), \psi_{n}(x)=\left(1-x^{2}\right)^{2} C_{n}(x)
$$

where $C_{n}(x)=\cos \left(n \cos ^{-1}(x)\right)$ is the $\mathrm{nth}$ order Chebyshev polynomial. $w(x, y, t), \quad \psi(x, y, t)$ and $T(x, y, t)$ are expanded in terms of $\phi_{n}(x)$ and $\psi_{n}(x)$ as

$$
\left.\begin{array}{l}
w(x, y, t)=\sum_{m=0}^{M} \sum_{n=0}^{N} w_{m n}(t) \phi_{m}(x) \phi_{n}(y), \\
\psi(x, y, t)=\sum_{m=0}^{M} \sum_{n=0}^{N} \psi_{m n}(t) \psi_{m}(x) \psi_{n}(y), \\
T(x, y, t)=\sum_{m=0}^{M} \sum_{n=0}^{N} T_{m n}(t) \phi_{m}(x) \phi_{n}(y)+x,
\end{array}\right\}
$$

where $M$ and $N$ are the truncation numbers in the $x$ and $y$ directions respectively. The expansion coefficients $w_{m n}, \psi_{m n}$ and $T_{m n}$ are then substituted into the basic Eqs. (2), (3) and (4) and the collocation method is applied. As

Research article

CIndian Society for Education and Environment (iSee)
Mondal et al. Indian J.Sci.Technol. 
a result, the nonlinear algebraic equations for $w_{m n}, \psi_{m n}$ and $T_{m n}$ are obtained. The collocation points are taken to be

$$
\left.\begin{array}{ll}
x_{i}=\cos \left[\pi\left(1-\frac{i}{M+2}\right)\right], & i=1, \ldots, M+1, \\
y_{i}=\cos \left[\pi\left(1-\frac{i}{N+2}\right)\right], & j=1, \ldots, N+1 .
\end{array}\right\}
$$

where $i=1, \ldots, M+1$ and $j=1, \ldots, N+1$. In the present numerical calculations, $M=20$ and $M=40$ have been used for sufficient accuracy of the solutions. The steady solutions are obtained by the Newton-Raphson iteration method. Linear stability of the steady solutions is investigated against two-dimensional ( $z$-independent) perturbations. To do this, the eigenvalue problem is solved which is constructed by the application of the function expansion method together with the collocation method to the perturbation equations obtained from Eqs. (2) to (4).

\section{Time-evolution Calculations}

In order to solve the non-linear time-evolution equations, we use the Crank-Nicolson and AdamsBashforth methods The Crank-Nicolson method is a finite difference method used for numerically solving the heat and related equations. For Navier-Stokes equations, it is frequently applied to the viscous and pressure gradient components. It is a second-order implicit time step method which calculates the average of $t(n)$ and $t(n+1)$ for all terms except for time differentials. This method has a good numerical stability as well. In order to maintain the numerical stability, it's been found that the time increment $\Delta t$ must be set below a certain limit value, and depending on the problem dealt with, this limit value changes. In this study, however, we use $\Delta t \leq 10^{-6}$.

For convenience in the discussion of the CrankNicolson method more explicitly, we consider the following one-dimensional heat-flow equation

$\frac{\partial q}{\partial t}=\sigma \frac{\partial^{2} q}{\partial x^{2}}$

where $q(t)$ is the temperature at time $t$ regarded as a functions of $x$ and $\sigma$ is the heat conductivity. The first time derivative in Eq. (12) is replaced by a finite difference ratio and the resulting ordinary differential equation with $x$ as an independent variable is integrated numerically and analytically. This integration is replaced for each finite time step. Considering a time step $\Delta t$, the derivative with respect to time may be written as:

$\frac{\partial q}{\partial t} \approx \frac{q(t+\Delta t)-q(t)}{\Delta t}$ as $\Delta t \rightarrow 0$.
Now taking the average at $t$ and $t+1$, Eq. (12) becomes approximately,

$$
\frac{q(t+\Delta t)-q(t)}{\Delta t}=\frac{\sigma}{2} \frac{\partial^{2}}{\partial x^{2}}[q(t+\Delta t)+q(t)] \text {. }
$$

The approximate solution of Eq. (12), thus evaluated, is a function of $\Delta t$ as well as $x$ and $t$, and the true solution is the limit of the approximate one as $\Delta t \rightarrow 0$. The method, determined by Eq. (14), is called the Crank-Nicolson method.

The Adams-Bashforth Method, on the other hand, is used for numerically solving initial value problems for ordinary differential equations. This method is an explicit linear multistep method that depends on multiple previous solution points to generate a new approximate solution point. In an Adams-Bashforth method of order $n$, the solution is advanced at each step by integrating the interpolant of the derivative values at $n$ previous points.

By applying the Crank-Nicolson method, as discussed above, to the non-dimensional basic equations (2) to (4), and rearranging, we get

$$
\begin{aligned}
\left(\frac{1}{\Delta t}-\frac{\Delta_{2}}{2}\right) w(t+\Delta t)= & \left(\frac{1}{\Delta t}+\frac{\Delta_{2}}{2}\right) w(t)-\delta x \frac{w(t)-w(t-\Delta t)}{\Delta t} \\
& +D_{n}+\delta x \Delta_{2} w(t)-\frac{\delta^{2} w(t)}{1+\delta x}+\delta \frac{\partial w(t)}{\partial x} \\
& +\frac{3}{2} \bar{P}(t)-\frac{1}{2} \bar{P}(t-\Delta t), \\
\left(\frac{1}{\Delta t}-\frac{\Delta_{2}}{2}\right) \Delta_{2} \psi(t+\Delta t)= & \left(\frac{1}{\Delta t}+\frac{\Delta_{2}}{2}\right) \Delta_{2} \psi(t)+\frac{\delta}{1+\delta x} \frac{1}{\Delta t}\left(\frac{\partial \psi(t)}{\delta x}-\frac{\partial \psi(t-\Delta t)}{\delta x}\right) \\
+\frac{\delta}{(1+\delta x)^{2}}\left\{-2(1+\delta x) \frac{\partial}{\delta x} \Delta_{2} \psi(t)+3 \delta \frac{\partial^{2} \psi(t)}{\delta x^{2}}-\frac{3 \delta^{2}}{(1+\delta x)} \frac{\partial \psi(t)}{\delta x}\right\} & -G r(1+\delta x) \frac{\partial T(t)}{\delta x}+\frac{3}{2} \bar{Q}(t)-\frac{1}{2} \bar{Q}(t-\Delta t) \\
\left(\frac{1}{\Delta t}-\frac{\Delta_{2}}{2 \operatorname{Pr}}\right) T(t+\Delta t)= & \left(\frac{1}{\Delta t}+\frac{\Delta_{2}}{2 \operatorname{Pr}}\right) T(t)-\delta x \frac{T(t)-T(t-\Delta t)}{\Delta t} \\
& +\frac{1}{\operatorname{Pr}} \delta\left(x \Delta_{2} T(t)+\frac{\partial T(t)}{\partial x}\right) \\
& +\frac{3}{2} \bar{R}(t)-\frac{1}{2} \bar{R}(t-\Delta t) .
\end{aligned}
$$

In the above formulations, $\bar{P}, \bar{Q}$ and $\bar{R}$ are the nonlinear terms. Then applying the Adams-Bashforth method for the second term of R.H.S of Eqs. (15), (16) \& (17) as:

$$
\begin{gathered}
-\delta x \frac{w(t)-w(t-\Delta t)}{\Delta t}=\frac{3}{2}\left(-\frac{2 \delta x}{\Delta t} w(t)-\frac{1}{2} \frac{2 \delta x}{\Delta t} w(t-\Delta t)\right)+\frac{2 \delta x}{\Delta t} w(t), \\
\frac{\delta}{1+\delta x} \frac{1}{\Delta t}\left(\frac{\partial \psi(t)}{\partial x}-\frac{\partial \psi(t-\Delta t)}{\partial x}\right)= \\
\frac{3}{2}\left(\frac{2 \delta x}{1+\delta x} \frac{1}{\Delta t} \frac{\partial \psi(t)}{\partial x}\right)-\frac{1}{2}\left(\frac{2 \delta x}{1+\delta x} \frac{1}{\Delta t} \frac{\partial \psi(t-\Delta t)}{\partial x}\right) \\
-\frac{2 \delta x}{1+\delta x} \frac{1}{\Delta t} \frac{\partial \psi(t)}{\partial x},
\end{gathered}
$$

and

$$
-\delta x \frac{T(t)-T(t-\Delta t)}{\Delta t}=\frac{3}{2}\left(-\frac{2 \delta x}{\Delta t} T(t)\right)-\frac{1}{2}\left(-\frac{2 \delta x}{\Delta t} T(t-\Delta t)\right)+\frac{2 \delta x}{\Delta t} T(t)
$$

Research article

CIndian Society for Education and Environment (iSee)
"Numerical analysis of flow in curved duct" http://www.indjst.org
Mondal et al. Indian J.Sci.Technol. 


$$
\begin{aligned}
&\left(\frac{1}{\Delta t}-\frac{\Delta_{2}}{2}\right) w(t+\Delta t)=D n+\left((1+2 \delta x)\left(\frac{1}{\Delta t}+\frac{\Delta_{2}}{2}\right)-\frac{\delta^{2}}{1+\delta x}+\delta \frac{\partial}{\partial x}\right) w(t) \\
&+\frac{3}{2} P(t)-\frac{1}{2} P(t-\Delta t) \\
&\left(\frac{1}{\Delta t}-\frac{\Delta_{2}}{2}\right) \Delta_{2} \psi(t+\Delta t)=\left\{\left(\frac{1}{\Delta t}+\frac{\Delta_{2}}{2}\right) \Delta_{2}-\frac{2 \delta}{1+\delta x} \frac{1}{\Delta t} \frac{\partial}{\partial x}\right. \\
&\left.-\frac{2 \delta}{1+\delta x} \frac{\partial}{\partial x} \Delta_{2}+\frac{3 \delta^{2}}{(1+\delta x)^{2}} \frac{\partial^{2}}{\partial x^{2}}-\frac{3 \delta^{3}}{(1+\delta x)^{3}} \frac{\partial}{\partial x}\right\} \psi(t) \\
&-G r(1+\delta x) \frac{\partial T(t)}{\partial x}+\frac{3}{2} Q(t)-\frac{1}{2} Q(t-\Delta t), \\
&\left(\frac{1}{\Delta t}-\frac{\Delta_{2}}{2 \operatorname{Pr}}\right) T(t+\Delta t)=\left\{(1+2 \delta x)\left(\frac{1}{\Delta t}+\frac{\Delta_{2}}{2 \operatorname{Pr}}\right)+\frac{1}{\mathrm{P}}\right. \\
&+ \frac{3}{2} R(t)-\frac{1}{2} R(t-\Delta t)
\end{aligned}
$$

where $P, Q$ and $R$ are also the non-linear terms expressed as,

$$
\begin{aligned}
& P=\widetilde{P}-\frac{2 \delta x}{\Delta t} w, \\
& Q=\widetilde{Q}+\frac{2 \delta}{1+\delta x} \frac{1}{\Delta t} \frac{\partial \psi}{\partial x} \\
& R=\widetilde{R}-\frac{2 \delta x}{\Delta t} T .
\end{aligned}
$$

By substituting the function expansion (10) into Eqs. (21), (22) and (23), and performing the numerical computations, we calculate $w(t+\Delta t), \psi(t+\Delta t)$ and

$T(t+\Delta t)$ for timeevolution calculation. Resistance coefficient

In the present study, the resistance coefficient $\lambda$ is used as the representative quantity of the flow state. It is also called the hydraulic resistance coefficient, and is generally used

Fig. 1. Coordinate system of the curved square duct

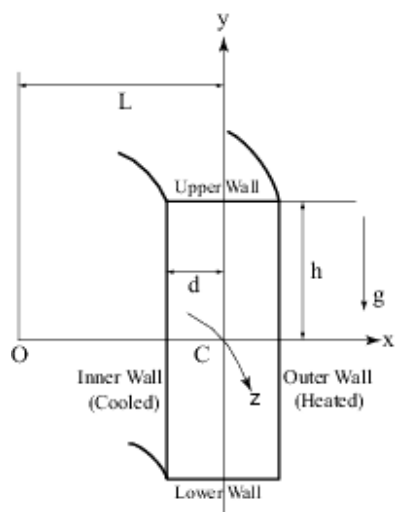

Research article CIndian Society for Education and Environment (iSee)
Fig. 2. (a) Steady solution branches for $\delta=0.1$ and $100 \leq D n \leq 1000$ for $G r=100$. (b) Enlargement of (a) at larger Dean numbers (675 $\leq$ Dn $\leq 1000$ )
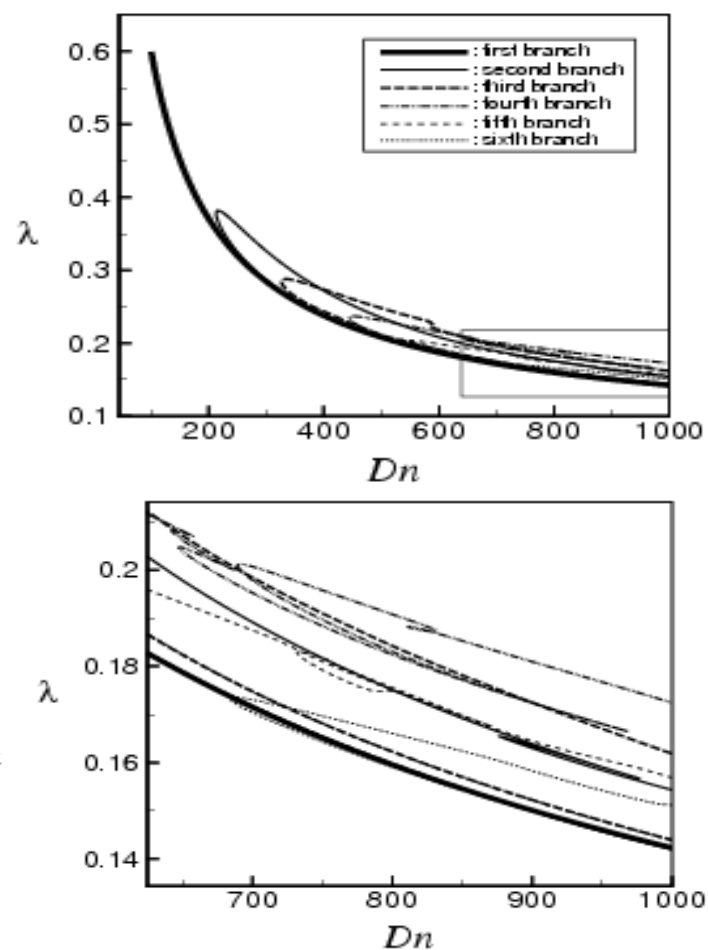

"Numerical analysis of flow in curved duct" http://www.indjst.org
Vol.2 No. 8 (Aug 2009)

ISSN: 0974- 6846

in fluid engineering, defined as

$\frac{P_{1}^{*}-P_{2}^{*}}{\Delta z^{*}}=\frac{\lambda}{d^{*}{ }_{h}} \frac{1}{2} \rho\left\langle w^{*}\right\rangle^{2}$

where quantities with an asterisk denote dimensional ones, \langle\rangle stands for the mean over the cross section of the rectangular duct, and $d_{h}{ }^{*}=4(2 d \times 2 d l) /(4 d+4 d l)$ is the hydraulic diameter. The mean axial velocity $\left\langle w^{*}\right\rangle$ is calculated by

$$
\left\langle w^{*}\right\rangle=\frac{v}{4 \sqrt{2 \delta d}} \int_{-1}^{1} d x \int_{-1}^{1} w(x, y, t) d y
$$

Since $\left(P_{1}^{*}-P_{2}^{*}\right) / \Delta z^{*}=G, \lambda$ is related to the mean nondimensional axial velocity $\langle w\rangle$ as

$$
\lambda=\frac{8 l \sqrt{2 \delta} D n}{(1+l)\langle w\rangle^{2}}
$$

where $\langle w\rangle=\sqrt{2 \delta} d\left\langle w^{*}\right\rangle / v$. In the present paper, $\lambda$ is used to denote the steady solution branches and to pursue the time evolution of the unsteady solutions.

dy solutions and their linear stability analysis

With the present numerical calculations, six branches of asymmetric steady solutions are obtained for the curvature, $\delta$, in the range $0<\delta \leq 0.5$ over a wide range of the Dean number, $D n$, $0 \leq D n \leq 1500$ for the fixed Grashof number $G r=100$. A bifurcation diagram, for example, is shown in Fig. 2(a) for $\delta=0.1$ and $100 \leq D n \leq 1000 \quad$ using $\lambda$, the representative quantity of the solutions. The steady solution branches are named as the first steady solution branch (first branch, thick solid line), the second steady branch (second branch, thin solid line), the third steady solution branch (third branch. long dashed line), the fourth steady solution branch (fourth branch, dash dot dot line), the fifth steady solution branch (fifth branch, dashed line), and the sixth steady solution branch (sixth branch, dotted line), respectively. In this regard, it should be remarked that Yanase et al. (2005) obtained five branches of steady solutions where the present fourth branch was absent. In order to observe the intricate branch structure as well as to distinguish the steady solution branches from each other, an enlargement about larger Dean numbers $(625 \leq D n \leq 1000)$ is shown in Fig. 2(b), where it is seen that the steady solution branches are independent and there exists no bifurcating relationship among them in the Mondal et al. Indian J.Sci.Technol. 
parameter

range

investigated in this paper.

The steady solution branches are obtained by the path continuation technique with various initial guesses as discussed by Mondal (2006). It is found that the first branch contains one- and two-vortex solutions. The second branch consists of two- and four-vortex solutions. The third branch is characterized by two- and four-vortex solutions, but different from the second branch in the form of vortices near the outer wall. The fourth branch contains two-, four-, and sixvortex solutions. The fifth branch is composed of two-, four-, six-, and eight-vortex solutions and the sixth branch two-, four-, six-, eight-, and ten-vortex solutions. It should be noted here that for other values of $\delta$, the bifurcation diagram is topologically unchanged from Fig. 2.

The first steady solution branch is solely depicted in Fig. for $10 \leq D n \leq 1500$. This is the only branch which exists throughout the whole range of $D n$ of the present study. To observe the change of the flow patterns and temperature distributions

Fig. 4. Stability criterion on the

$D n-\delta$ plane (circle with broken line: boundary between the stable and unstable solution regions)

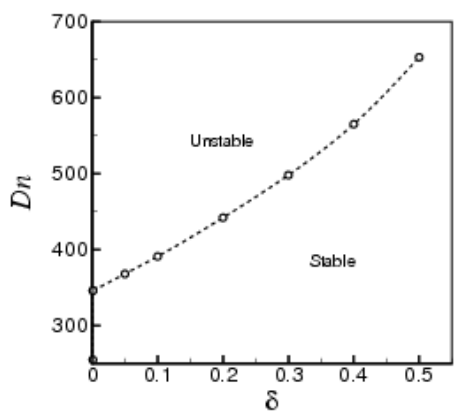

Research article

(C)Indian Society for Education and Environment (iSee)
Fig.3. (a) First steady solution branch with the region of linear stability (bold line). (b) Contours of secondary flow (top) and temperature profile (bottom) on the first steady solution branch for

$\delta=0.1$ and $0<D n \leq 1500$
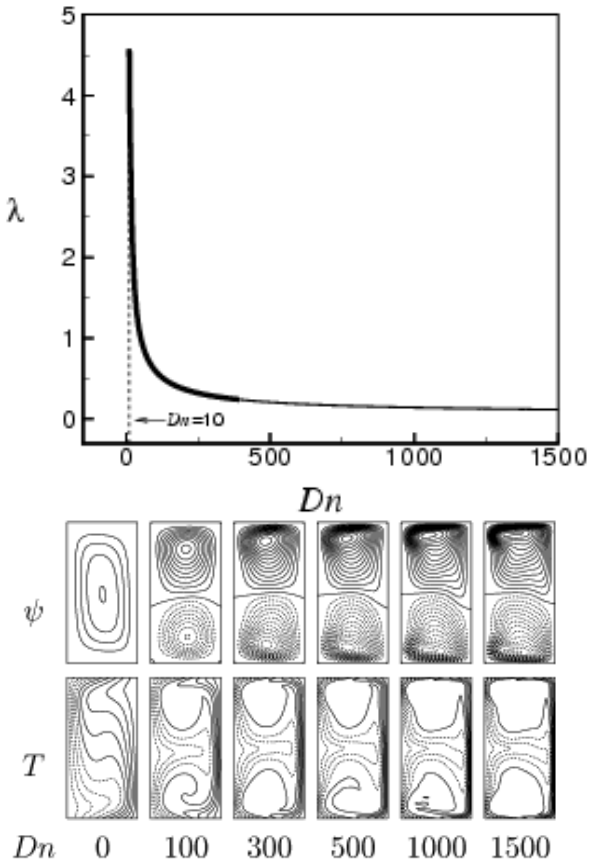

Fig. 5. The results for $\delta=0.1$ and $D n=425$. (a)

Time evolution of $\lambda$ and the values of $\lambda$ for the steady solutions for $0 \leq t \leq 20$. (b) Contours of secondary flow (top) and temperature profile (bottom) for one period of oscillation at $16.75 \leq t \leq 17.35$
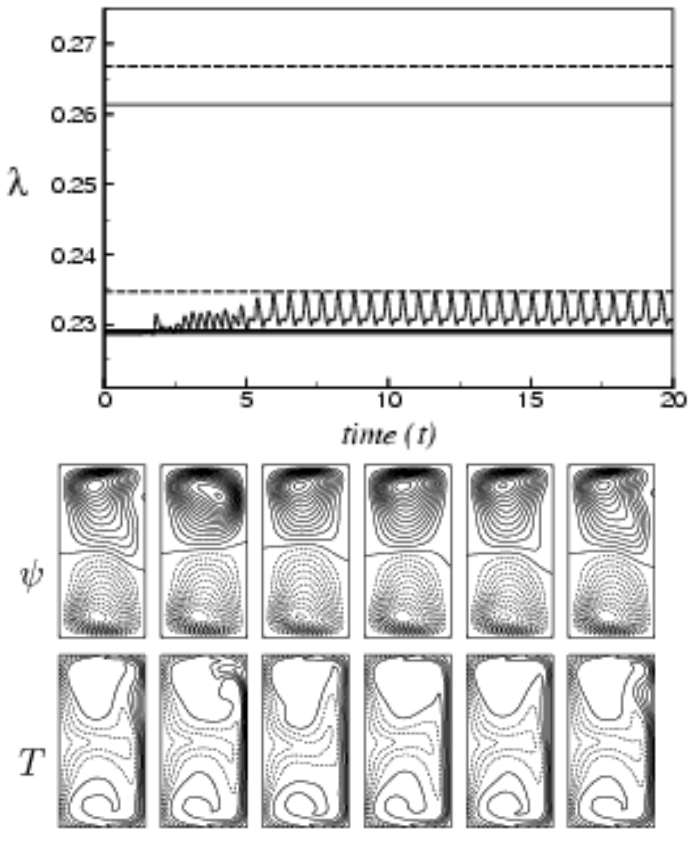

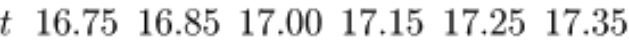
"Numerical analysis of flow in curved duct" http://www.indjst.org
Vol.2 No. 8 (Aug 2009)

ISSN: 0974- 6846

contours of typical secondary flow and temperature profile at several values of $D n$ are shown in Fig. 3(b), where the contours of $\psi$ and $T$ are drawn with the increments $\Delta \psi=0.6$ and $T=0.2$, respectively. The same increments of $\psi$ and $T$ are used for all the figures in this paper, if not specified. Righthand side of each box is in the outside direction of the duct curvature. In the figures of the secondary flow, solid lines (positive $\psi$ ) show that the secondary flow is in the counter clockwise direction while the dotted lines (negative $\psi$ ) are in the clockwise direction. In the figures of the temperature field, solid lines are those for $T \geq 0$ and dotted ones for $T<0$. As seen in Fig. 3(b), the first steady solution branch consists of one- and twovortex solutions which are asymmetric with respect to the horizontal centre plane $y=0$. Heating the outer wa1l causes deformation of the secondary flow and yields asymmetry of the flow.

Linear stability of the steady solutions is then investigated. It is found that among six branches of steady solutions, only the first branch is linearly stable in a single interval of $D n$ for any $\delta$ in the range, while the other branches are linearly unstable at any values of $D n$ or $\delta$. The stability result for $\delta=0.1$, for example, is shown in Table 1, where the eigenvalues with the maximum real part of $\sigma$ are listed; those for the linearly stable solutions are printed in hold letters. As seen in Table 1, the stability region for $\delta=0.1$ exists for $0 \leq D n \leq 391.792 \quad$ and the

Fig. 6. Power-spectrum of the time evolution of $\lambda$ for $D n=425$ at $\delta=0.1$

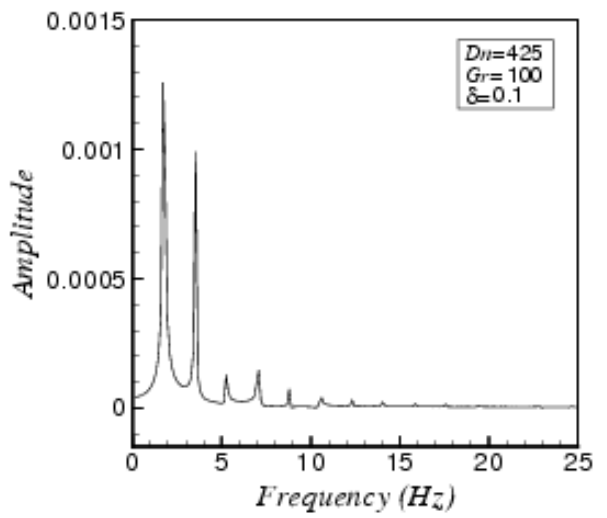

Mondal et al. Indian J.Sci.Technol. 
perturbation grows oscillatorily for $D n \geq 391.793$. The total stability characteristics, however, is illustrated in Fig. 4. In this figure, the regions of stable and unstable solutions are separated by the circles and broken lines where the circles denote stable steady solutions on the boundary calculated in this study. As seen in Fig. 4, the stability region increases monotonically as $\delta$ increases gradually.

\section{Time evolution and spectral analysis}

In order to study the nonlinear behavior of the unsteady solutions, time evolution calculations as well as their spectral analysis are performed for $0<\delta \leq 0.5$. In Sec. 6.2.1, the results of the time evolution calculations as well as the spectral analysis are discussed in detail, for $\delta=0.1$ at different values of $D n$ and those for other values of $\delta$ in Sec. 6.2.2, which cover the whole range of $\delta$ investigated in this paper.

Time evolution and spectral analysis for $\delta=0.1$ : Time evolutions of $\lambda$ for $D n \leq 391$ and $\delta=0.1$, at which the steady solution is linearly stable on the first branch, show that the value of $\lambda$ quickly approaches that of the stable solution on the first branch. Then in order to see the unsteady flow characteristics obtained for $D n \geq 392$, where the solution is linearly unstable on the first branch, time evolution calculations are performed at several values of $D n$. Figure 5(a) shows time evolution result for $D n=425$, where it is seen that the flow oscillates multi-periodically. In the same figure, to observe the relationship between the periodic solution and the steady states, the values of $\lambda$ for the steady solution branches at $D n=425$ are also shown by straight lines using the same kind of lines as were used in the bifurcation diagram in Fig. 2. As seen in Fig. 5(a), the periodic solution at $D n=425$ oscillates in the region above the first but below the third steady solution branches. It shows that the first branch and the lower part of the third branch play a role of an envelope of this periodic oscillation. To observe the periodic change of the flow patterns and temperature distributions, contours of secondary flow and temperature profiles are shown in Fig. 5(b) for one period of oscillation at $16.75 \leq t \leq 17.35$, where it is seen that the multi-periodic oscillation at $D n=425$ is a two-vortex solution. In this regard, it

should be noted that we obtained only periodic solutions for $392 \leq D n \leq 425$.

Now in order to investigate the transition from the periodic solution to the chaotic one, the power spectrum of the time change of $\lambda$ is calculated. The power spectrum for $D n=425$ is shown in Fig. 6. In this figure, it is found that not only the line spectrum of the fundamental frequency $(f=1.92 \mathrm{~Hz})$ and its harmonics but also the other line spectrum $(f=7.0 \mathrm{~Hz})$ and its harmonics are seen. This result clearly shows that the oscillation presented in Fig. 5(a) is multi-periodic. If $D n$ is increased further, the periodic oscillation turns into a chaotic oscillation via multi-periodic oscillation, and this transition is clearly identified by the power spectrum analysis.

Time evolution of $\lambda$ is then performed for $D n=435$ as shown in Fig. 7(a). In this figure, the values of $\delta$ for the steady solution branches are also plotted by straight lines. As seen in Fig. 7(a), the flow oscillates irregularly with the large windows of quasi-periodic oscillations which suggest that the flow is chaotic then. It is found that, the chaotic solution at $D n=435$ fluctuate around

Fig.7. The results for $\delta=0.1$ and $D n=435$. (a) Time evolution of $\lambda$ and the values of $\lambda$ for the steady solutions for $0 \leq t \leq 40$. (b) Contours of secondary flow (top) and temperature profile (bottom) for $30.0 \leq t \leq 32.5$
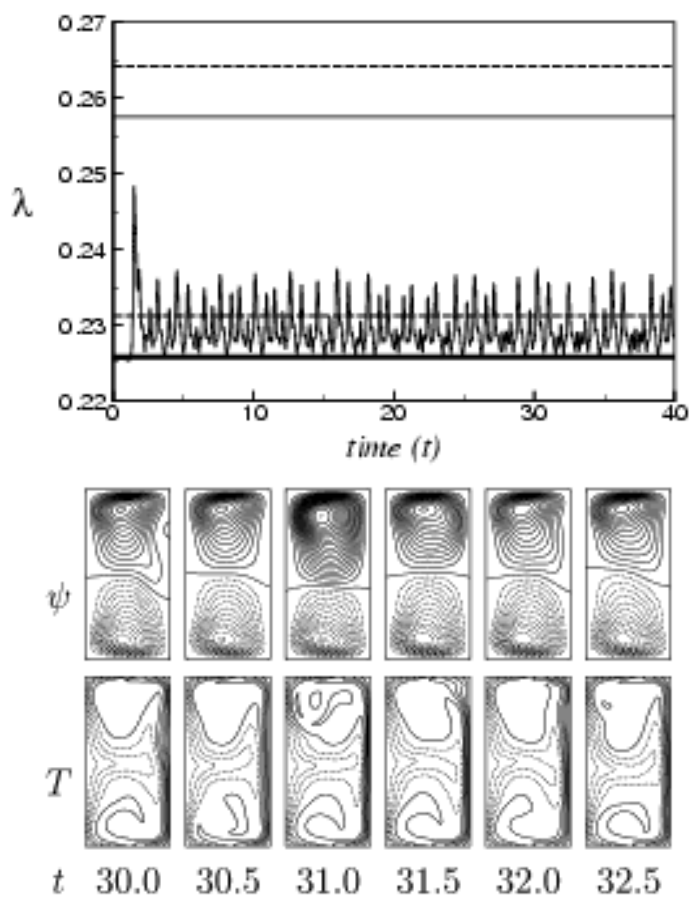

the value of $\lambda$ on the lower branch of the third steady solution at $D n=435$, and the first branch seems to be an lower trajectory of this chaotic oscillation. Contours of secondary flow and temperature profile, shown in Fig. 7(b) for $D n=435$ at $30.0 \leq t \leq 32.5$, show that the chaotic oscillation at $D n=435$ is a two-vortex solution. The power spectrum for $D n=435$ is then performed as shown in Fig. 8. It is found that as $D n$ increases, alternating occurrence of the oscillating and chaotic states are observed as shown in Fig. 7(a) for $D n=435$. The corresponding power spectrum shows that it consists of the line spectrum with nearly the same frequency as of $D n=425$ and a continuous spectrum. It is clear that the former corresponds to the periodic state and the latter to the chaotic one. Figure 8 clearly shows that the unsteady flow
Research article

CIndian Society for Education and Environment (iSee)
"Numerical analysis of flow in curved duct" http://www.indjst.org
Mondal et al. Indian J.Sci.Technol. 
at $D n=435$ is a chaotic solution. We may thus, by the power spectrum results shown in Figs.7-8, conclude that the transition from periodic to chaotic state occurs between $D n=425$ and $D n=435$.

Next, time evolutions of $\lambda$ together with the values of $\lambda$ for the steady solution branches, indicated by straight lines, are shown in Figs. 9(a) and $10(\mathrm{a})$ for $D n=1000$ and 1500 respectively, where it is seen that the flow is chaotic for both the cases. It is observed from Fig. 9(a) that the unsteady solution at $D n=1000$ drifts in the region between the third and fourth steady solution branches while that for $D n=1500$ (Fig. 10(a)) fluctuates above all the steady solution branches and the upper part of the fourth branch, which has the maximum $\lambda(\lambda=0.1352701)$ at $D n=1500$, looks like an envelope of this chaotic solution. The chaotic solution for $D n=1000$ is called a weak chaos, and that for $D n=1500$ strong chaos (Mondal et al., 2007), since the chaotic solution at $D n=1000$ is still trapped by the steady solution branches but that for $D n=1500$ tends to get away from them. In order to observe the change of the flow patterns and temperature distributions, contours of secondary flow and temperature profiles for $D n=1000$ and $D n=1500$ are shown in Figs. 9(b) and 10(b) respectively, where the increments $\Delta \psi=1.2$ and $\Delta T=0.4$ are used to draw the contours of secondary flow and temperature profile respectively, As seen in the secondary flow patterns, the chaotic solution at $D n=1000$ and $D n=1500$ is composed of two- and multi-vortex solutions. In this paper, the power spectra for other values of $D n$ are not shown for brevity but only for $D n=425$ and $D n=435$. The reason
Fig.9. The results for $\delta=0.1$ and $D n=1000$. (a) Time evolution of $\lambda$ and the values of $\lambda$ for the steady solutions for $0 \leq t \leq 10$. (b) Contours of secondary flow (top) and temperature profile

(bottom) for $5.0 \leq t \leq 7.5$

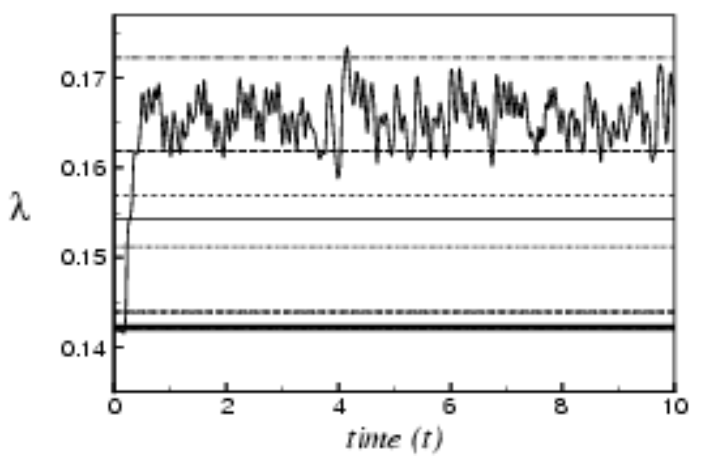

$\psi$

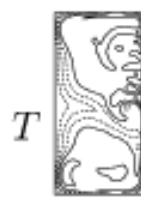

$t \quad 5.0$ the $D n-\delta:$ In this subsection, the distribution of the steady, periodic and chaotic solutions, obtained by the time evolution computations, is presented in Fig. 11 in the Dean number versus curvature $(D n-\delta)$ plane for $0 \leq D n \leq 1500$ and $0 \leq \delta \leq 0.5$. In this figure, the circles indicate stable steady solutions, squares periodic solutions and triangles chaotic solutions. As seen in Fig. 11 , steady flows turn into chaotic flows through periodic or multi-periodic flows, if $D n$ is increased no matter what $\delta$ is. It is observed that the periodic solution occurs in a small region of $D n$ for any $\delta$, and as $\delta$ becomes large, the region of stable-steady solution increases monotonically and consequently the occurrence of the periodic state and hence the chaotic state is delayed as $D n$ is increased.

Conclusions

In this paper, a detailed numerical study of the nonisothermal flow through a curved rectangular duct of aspect ratio 2 has been

performed by using the
ing a wide range of the Dean is that we want to show the transitional behavior of the unsteady solutions as well as the Dean number where the transition occurs as the flow turns into chaotic from periodic in this region. In this study, the time evolution calculations for other values of $\delta$ show that the timedependent behavior is similar to that of $\delta=0.1$. spectral method, and covering a wide range of the Dean
number $0 \leq D n \leq 1500$ and the curvature $0 \leq \delta \leq 0.5$ for the Grashof number $G r=100$.

After a comprehensive survey over the parametric ranges, six branches of asymmetric steady solutions are obtained for any $\delta$ laying the range. Linear stability of
Research article

CIndian Society for Education and Environment (iSee)
"Numerical analysis of flow in curved duct" http://www.indjst.org
Mondal et al. Indian J.Sci.Technol. 
the steady solutions shows that only one branch is linearly stable while other branches are linearly unstable. Time evolution calculations as well as their spectral analyses show that the steady flow turns into chaos through periodic and multi-periodic flows in the scenario "steady - periodic - chaotic", if $D n$ is increased no matter what $\delta$ is. It is found that the larger the curvature is, the larger becomes the region of stable steady solution on the first branch. The periodic solution occurs in a small region of $D n$ where the stability disappears. The occurrence of the periodic state and hence the chaotic state is delayed as $\delta$ increases. In order to investigate the transition from the multiperiodic oscillations to chaotic states in more detail, the spectral analysis is found to be very useful. In this regard, it is interesting to note that the chaotic solution is weak for smaller $D n$ 's, where the solution is trapped by the steady solution branches; for larger $D n$ 's, on the other hand, the chaotic solution becomes strong, where the solution gets away from the steady solution branches.

\section{References}

1. Berger SA, Talbot $L$ and Yao LS (1983) Flow in curved pipes. Annu. Rev. Fluid. Mech. 35, 461- 512.

2. Chandratilleke TT and Nursuhyakto (2003) Numerical prediction of secondary flow and convective heat transfer in externally heated curved rectangular ducts. Int. J. Thermal Sci. 42, 187-198.

3. Dennis SCR and $\mathrm{Ng} \mathrm{M}$ (1982) Dual solutions for steady laminar flow through a curved tube. Quart. J. Mech. Appl. Math. 35, 305 - 324.

4. Ito $\mathrm{H}$ (1987) Flow in curved pipes. JSME Int. J. 30, 543-552.

5. Mondal RN (2006) Isothermal and nonisothermal flows through curved ducts with square and rectangular cross sections, Ph. D. Thesis, Dept. of Mech. Engg., Okayama Univ., Japan.

6. Mondal RN, Kaga $\mathrm{Y}$, Hyakutake $\mathrm{T}$ and Yanase $\mathrm{S}$ (2006) Effects of curvature and convective heat transfer in curved square duct flows. Trans. ASME J. Fluids Engg. 128, 1013-1022.

7. Mondal RN, Kaga $Y$, Hyakutake $T$ and Yanase $S$ (2007) Bifurcation diagram for two-dimensional steady flow and unsteady solutions in a curved square duct. Fluid Dyn. Res. 39, 413-446.

Vol.2 No. 8 (Aug 2009)

ISSN: 0974- 6846

Fig. 10. The results for $\delta=0.1$ and $D n=$ 1500. (a) Time evolution of $\lambda$ and the values of $\lambda$ for the steady solutions for $0 \leq t \leq 10$. (b) Contours of secondary flow (top) and temperature profile (bottom) for $6.0 \leq t \leq 8.0$
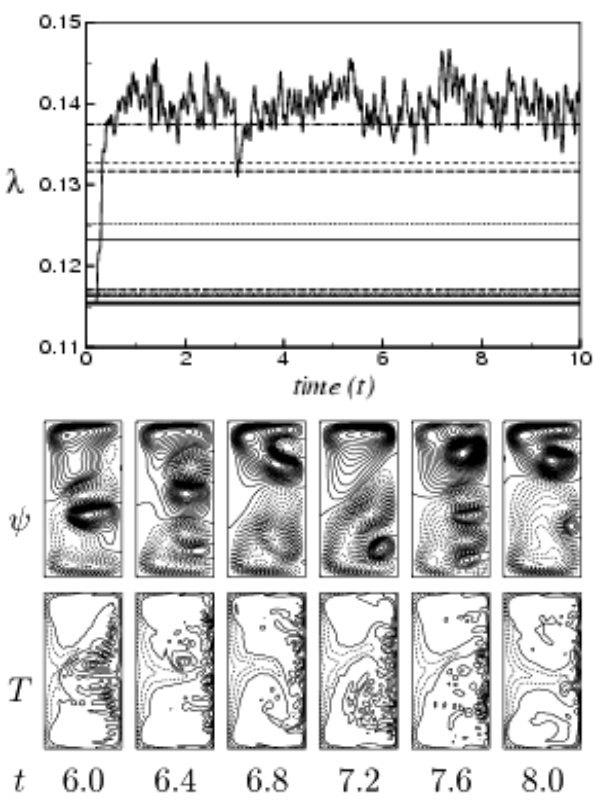

Fig. 11. Distribution of the time-dependent solutions in the $D n-\delta$ plane for

$0 \leq D n \leq 1500$ and $0<\delta \leq 0.5$ for $G r$ $=100(\mathrm{O}:$ Steady-stable solution, $\square$ :

Periodic solution. $\Delta$ : Chaotic solution).

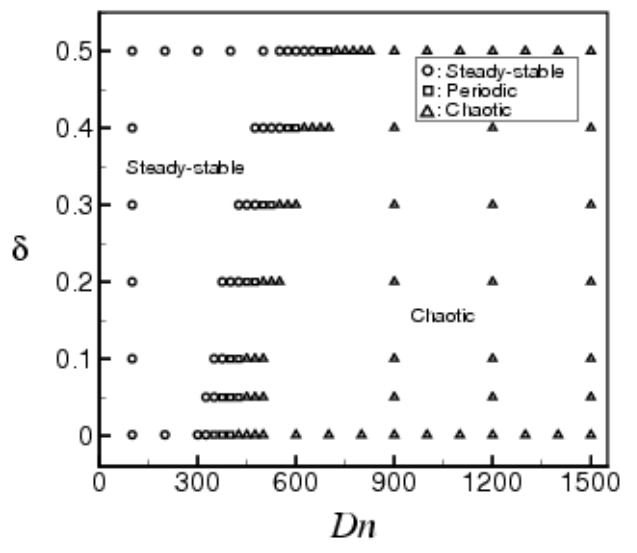

8. Nandakumar $\mathrm{K}$ and Masliyah JH (1986) Swirling flow and heat transfer in coiled and twisted pipes. Adv. Transport. Process. 4, 49112.

9. Wang $L$ and Yang $T$ (2005) Periodic oscillation in curved duct flows, Physica D. 200, 296-302.

10. Winters KH (1987) A bifurcation study of laminar flow in a curved tube of rectangular cross-section. $J$. Fluid Mech. 180, 343- 369.

11. Yanase $S$ and Nishiyama $K$ (1988) On the bifurcation of laminar flows through a curved rectangular tube. J. Phys. Soc. Japan. 57, 37903795.

12. Yanase S, Goto $N$ and Yamamoto K (1989) Dual solutions of the flow through a curved tube. Fluid Dyn. Res. 14, 191- 201.

13. Yanase $S$, Kaga $Y$ and Daikai R (2002) Laminar flows through a curved rectangular duct over a wide range of the aspect ratio. Fluid Dyn. Res. 31, 151-183.

14. Yanase S, Mondal RN, Kaga $\mathrm{Y}$ and Yamamoto $\mathrm{K}$ (2005) Transition from steady to chaotic states of isothermal and nonisothermal flows through a curved rectangular duct. J. Phys. Soc. Japan. 74, 345- 358.

15. Yanase $S$, Yamamoto $K$ and Yoshida T (1994) Effects of curvature on dual solutions of flow through a curved circular tube. Fluid Dyn. Res. 13, 217-228.

16. Yang $Z$ and Keller HB (1986) Multiple laminar flows through curved pipes. Appl. Numr. Maths. 2, 257271.
Research article

CIndian Society for Education and Environment (iSee)
"Numerical analysis of flow in curved duct" http://www.indjst.org
Mondal et al. Indian J.Sci.Technol. 